\section{Long-term efficacy of infliximab on background vascular leakage in patients with Behçet's disease}

H Keino, AA Okada, T Watanabe and W Taki
Department of Ophthalmology, Kyorin University School of Medicine, Tokyo, Japan

Correspondence: AA Okada, Department of Ophthalmology, Kyorin University School of Medicine, 6-20-2 Shinkawa, Mitaka, Tokyo 181-8611, Japan Tel: +81 422475511 ; Fax: +81422469309. E-mail: aokada@ eye-center.org

Received: 22 October 2013 Accepted in revised form: 11 May 2014 Published online: 20 June 2014

\begin{abstract}
Purpose To evaluate the effect of infliximab over the initial 4 years of treatment on inflammatory ocular attacks and background retinal/disc vascular leakage in patients with refractory uveoretinitis associated with Behçet's disease.

Methods Clinical records of nine patients were retrospectively reviewed. The main outcomes analyzed were frequency of ocular inflammatory attacks, background retinal and disc vascular leakage as assessed by fluorescein angiography during periods of clinical quiescence, best-corrected visual acuity, and adverse effects.

Results The median follow-up on infliximab was 50 months (range 48-58 months). Mean frequency of attacks decreased significantly in years 1, 2, 3, and 4 compared with the baseline 1-year period before infliximab use. Mean background retinal and disc vascular leakage scores also decreased significantly at the end of each 1-year period compared with baseline. Visual acuity improved or was unchanged at the end of 4 years in 17 of 18 eyes. No serious adverse effects were observed.

Conclusion Infliximab reduced the mean frequency of ocular attacks and mean background retinal/disc vascular leakage in a long-term sustained manner over 4 years of treatment in Behçet's disease patients.

Eye (2014) 28, 1100-1106; doi:10.1038/eye.2014.138; published online 20 June 2014
\end{abstract}

\section{Introduction}

Behçet's disease is a systemic inflammatory disease characterized by recurrent oral aphthous ulcers, uveitis, cutaneous manifestations, and genital ulcers. ${ }^{1-3}$ Although the etiology of this disorder remains unknown, both genetic and environmental factors appear to be important in its pathogenesis. ${ }^{4}$ Patients with ocular involvement experience unilateral or bilateral acute episodes of anterior uveitis with or without hypopyon, posterior uveitis, and/or panuveitis. ${ }^{1-3}$ A recent report suggested that a higher frequency of ocular attacks is significantly associated with poorer visual outcome. ${ }^{5}$

Background retinal and disc vascular leakage can be observed by fluorescein angiography (FA) during periods of clinical quiescence. ${ }^{6,7} \mathrm{We}$ believe that severity grading of FA leakage may serve as a useful surrogate marker for the evaluation of intraocular inflammatory activity level in patients with Behçet's disease.

Biologic agents such as infliximab, an antitumor necrosis factor- $\alpha$ antibody, have demonstrated good efficacy for the treatment of ocular manifestations of Behçet's disease..$^{8-12}$ A recent prospective multicenter study showed that the uveoretinitis improved or improved somewhat in over $90 \%$ of patients, with a corresponding increase in mean visual acuity. ${ }^{13}$ Furthermore, we have previously reported that infliximab significantly reduced vascular leakage in the peripheral retina as assessed by FA, as well as frequency of ocular attacks after 12 months of therapy. ${ }^{14}$

Here we report the efficacy and safety of infliximab over a long term of treatment in patients with ocular Behçet's disease. Efficacy was assessed by evaluating frequency of observed ocular inflammatory attacks and degree of background retinal and disc vascular leakage on FA.

\section{Materials and methods}

\section{Patients}

Medical records were reviewed of nine patients with Behçet's disease followed in the Ocular 
Inflammation Service at the Kyorin Eye Center, Kyorin University Hospital (Tokyo, Japan) who received infliximab therapy for at least 4 years between January 2007 and March 2012. Diagnosis was based on the criteria of the Behçet's Disease Research Committee of Japan. ${ }^{15}$ As per Japanese Ministry of Health, Labor, and Welfare (MHLW) guidelines concerning the indication for initiating infliximab therapy, all patients had ocular inflammation refractory to treatment with at least one immunosuppressive drug and/or corticosteroids, or were judged to be intolerant to such therapy due to adverse effects.

\section{Treatment protocol}

Before starting infliximab, all patients underwent a complete internal medical examination, tuberculin protein purified derivative (PPD) skin testing, and chest X-ray. Patients found to be PPD-positive received tuberculosis prophylaxis consisting of $400 \mathrm{mg} /$ day of isoniazid for at least 6 months. Infliximab treatment was initiated as per the MHLW-approved dosing of $5 \mathrm{mg} / \mathrm{kg}$ administered intravenously at weeks $0,2,6$, and every 8 weeks thereafter. In patients receiving cyclosporine at the time of initiating infliximab therapy, the cyclosporine was tapered down to a lower dose, generally $100 \mathrm{mg} /$ day, and maintained in the attempt to inhibit development of human anti-chimera antibodies (HACA). ${ }^{16,17}$

\section{Main outcome measures}

The main outcome measures were frequency of observed ocular inflammatory attacks, background retinal and disc vascular leakage assessed by FA during periods of clinical quiescence, best-corrected visual acuity (BCVA), and adverse effects.

Anterior chamber cells and flare were graded as per the SUN criteria. ${ }^{18}$ Ocular inflammatory attacks were defined as the sudden onset of cells and/or flare in the anterior chamber or vitreous, or cellular infiltrates and/ or hemorrhage in the retina, as observed clinically by slitlamp biomicroscopy and/or indirect funduscopy. Number of ocular attacks before and after initiating infliximab treatment was converted to frequency per 12-month period. To assess the degree of background retinal and disc vascular leakage, FA was performed before initiation of infliximab therapy (baseline) and at the end of years 1, 2, 3, and 4, all at times of clinical quiescence defined as the absence of signs of an ocular inflammatory attack. The extent of fluorescein leakage was graded on a scale of $0-3(0=$ none, $1=$ mild, $2=$ moderate, $3=$ severe) for the peripheral retina, the macula, and the optic disc by two uveitis specialists (HK and AAO) in a masked fashion. ${ }^{14}$ Mean scores were calculated for the peripheral retina, macula, and optic disc separately, with the total vascular leakage score representing the sum of these three mean scores. ${ }^{14}$

BCVAs were measured at each clinic visit. Adverse events including infusion reactions (occurring during and up until $2 \mathrm{~h}$ after conclusion of the infliximab infusion) were recorded. This study was conducted in accordance with the tenets of the Declaration of Helsinki, and was deemed by the Kyorin University Hospital Research Ethics Committee not to require institutional review board approval. All applicable institutional and governmental regulations concerning the ethical use of human volunteers/animals were followed during this research.

\section{Statistical analysis}

Statistical analysis was performed using the Wilcoxon signed-rank test. A $P$-value $<0.05$ was considered to be statistically significant. All results were expressed as mean $\pm S E$.

\section{Results}

The demographical characteristics of the nine patients included in the study are summarized in Table 1. All the patients were men, ranging in age from 13 to 68 years (mean 35 years). The median duration of intraocular inflammation associated with Behçet's disease before starting infliximab therapy was 30 months. Manifestations or history of oral aphthous ulcers, skin lesions, and genital ulcers were noted in 9, 6, and 4 patients, respectively. Systemic treatment at the time of starting infliximab included cyclosporine in five patients, cyclosporine plus prednisolone in two patients, and cyclosporine, prednisolone plus methotrexate in one patient. After initiation of infliximab therapy, the cyclosporine was tapered in the eight patients who were on this drug, and the prednisolone and methotrexate were discontinued in all patients.

The median follow-up on infliximab therapy was 50 months (range 48-58 months). As shown in Figure 1a, the mean frequency of ocular attacks/year was significantly reduced in year 1 on infliximab compared with the 1-year period before starting infliximab $(P=0.0425)$. This mean frequency remained stable and was significantly decreased compared with baseline in year $2(P=0.0277)$, year $3(P=0.0178)$, and year $4(P=0.0273)$. Figure $1 \mathrm{~b}$ shows the frequency of ocular attacks for individual patients. Although the number of ocular attacks remained low in most patients over the 4 years, one patient (patient 5) showed a trend towards rising frequency over time. In this patient, the frequency of ocular attacks began to rise in year 2 compared with year 1 ; weakening of efficacy was suspected in this case, 
Table 1 Baseline patient demographics and clinical manifestations

\begin{tabular}{lc}
\hline Total number of patients & 9 \\
Gender & \\
Male & 9 \\
Female & 0 \\
Age (years) & \\
Mean & 35 \\
Range & $13-68$ \\
Duration of ocular disease (months) & \\
Median & 30 \\
Range & $13-105$ \\
Systemic manifestations (no. of patients) & \\
Oral aphthous ulcers & 9 \\
Skin lesions & 6 \\
Genital ulcers & 4 \\
Therapy at baseline (no. of patients) & \\
Cyclosporine & \\
Prednisolone & \\
Methotrexate & 3 \\
\hline
\end{tabular}

and thus the interval between infliximab infusions was shortened from 8 to 6 weeks.

Figure 1c shows the mean total vascular leakage score as assessed by FA. There was a significant decrease in the mean leakage score compared with baseline at the end of year $1(P=0.0112)$, year $2(P=0.0074)$, year $3(P=0.0074)$, and year $4(P=0.00112)$. The mean vascular leakage score was lowest at the 2-year mark. Figure 1d demonstrates the vascular leakage score of each patient before and after initiating infliximab therapy. Although individual total vascular leakage scores were reduced in all patients for the first 2 years of infliximab therapy, three patients (patients 2, 5, and 8) showed a mild trend toward rising scores after 3 years of treatment.

The visual outcomes of infliximab therapy are shown in Figure 2. The BCVA was maintained or improved in all 18 eyes of 9 patients at the end of year 1 (Figure 2a), and in 17 of 18 eyes (94\%) at the end of year 4 (Figure 2b). Two eyes $(11 \%)$ that had a BCVA of 0.1 or less at baseline had optic disc atrophy and/or macular atrophy before the initiation of infliximab therapy, and experienced no improvement in visual acuity. One eye (in patient 9)
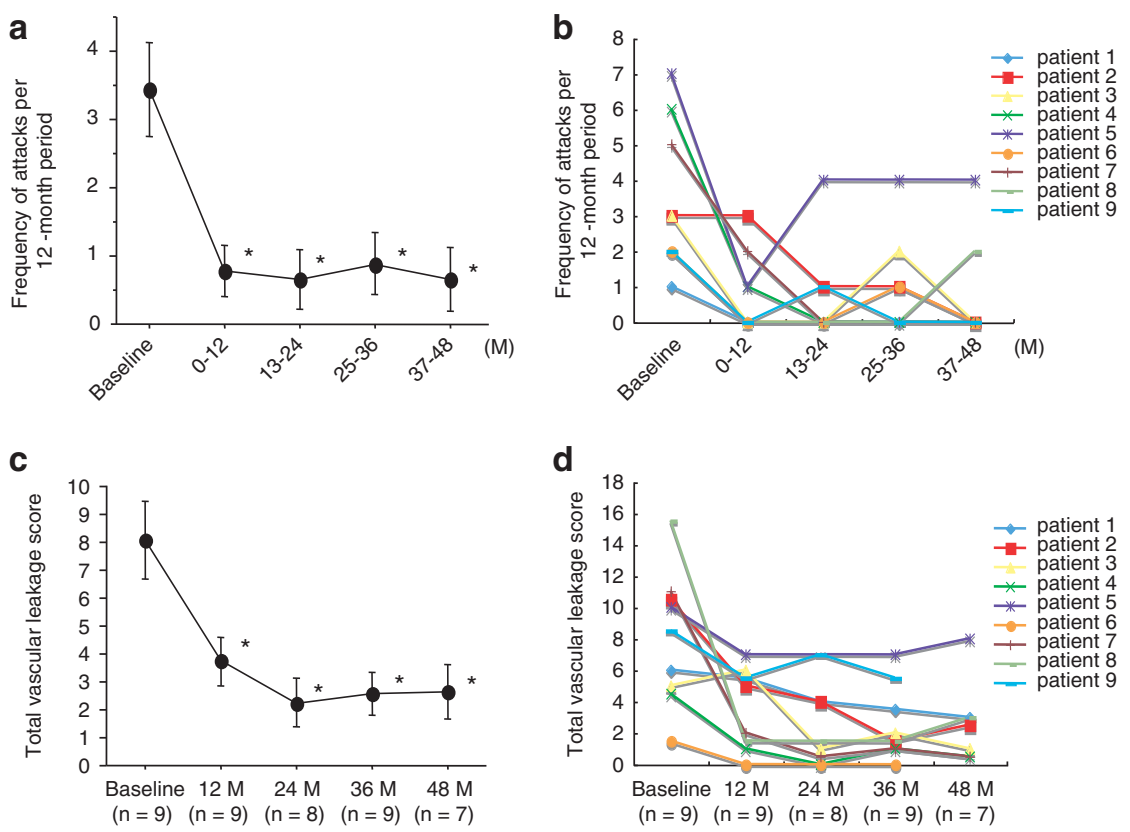

Figure 1 Frequency of ocular inflammatory attacks and fluorescein vascular leakage scores before and after initiation of infliximab therapy. (a) The number of ocular inflammatory attacks before and after initiation of infliximab treatment was converted to frequency/ year. The mean $\pm S E$ for all patients is shown for each time point. Statistical analysis was performed using the Wilcoxon signed-rank test. ${ }^{*} P<0.05$ versus baseline. (b) Frequency of ocular attacks/year for individual patient over each 1-year period. (c) To assess degree of vascular leakage, FA was performed at times of clinical quiescence before the initiation of infliximab therapy (baseline) and at the end of years $1,2,3$, and 4 on infliximab. Degree of fluorescein leakage was graded on a scale of $0-3(0=$ none, $1=$ mild, $2=$ moderate, 3 = severe) for the optic disc, the macula, and the peripheral retina of each study eye by two masked graders, and averaged. The total vascular leakage score represents the sum of the averages for the disc, macula and peripheral retina. The mean \pm SE for all patients is shown at each time point. Statistical analysis was performed using the Wilcoxon signed-rank test. ${ }^{*} P<0.05$ versus baseline. (d) Total vascular leakage scores are shown for individual patients at baseline and at the end of years 1,2,3, and 4 on infliximab. $\mathrm{M}=$ months. 
experienced a decrease in BCVA from 0.8 at baseline to 0.4 at the end of 4 years; this was believed to be due to progressive optic disc atrophy.

With regard to adverse effects, five patients experienced symptoms consistent with the common cold at some point during the 4 years or more of infliximab therapy; each episode resolved without specific treatment. In addition, one patient developed bacterial pharyngitis that responded to antibiotic therapy. Two patients experienced
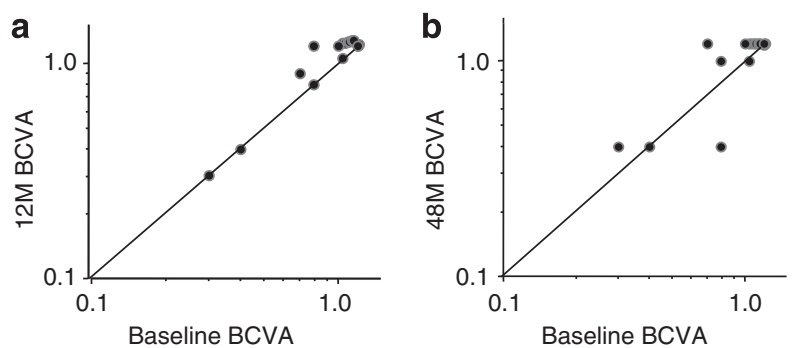

Figure 2 Visual acuities at baseline and on infliximab therapy. BCVA of all study eyes are shown at baseline and at the end of 1 year (a) and 4 years (b) after initiation of infliximab therapy. $\mathrm{M}=$ months. infliximab-related infusion reactions consisting of rash and itchiness, occurring during the 15th infusion in patient 3 and the 8 th infusion in patient 9 . Both patients had been receiving concomitant cyclosporine. After the initial infusion reaction, subsequent infusions were performed with pre-administration of intravenous hydrocortisone $(100 \mathrm{mg}$ ) and the infusion reactions abated in both patients. None of the adverse effects observed, including the infusion reactions, met MHLW criteria for being serious adverse effects.

\section{Representative case reports}

\section{Patient 5}

This patient was a 21-year-old Japanese man who had been diagnosed in January 2007 with recurrent uveoretinitis due to Behçet's disease. Despite treatment with colchicine and cyclosporine, the patient continued to have posterior ocular inflammatory attacks in both eyes, and infliximab therapy was started in November 2007. Over the next 6 months, the colchicine was discontinued and the cyclosporine was reduced

\section{a}
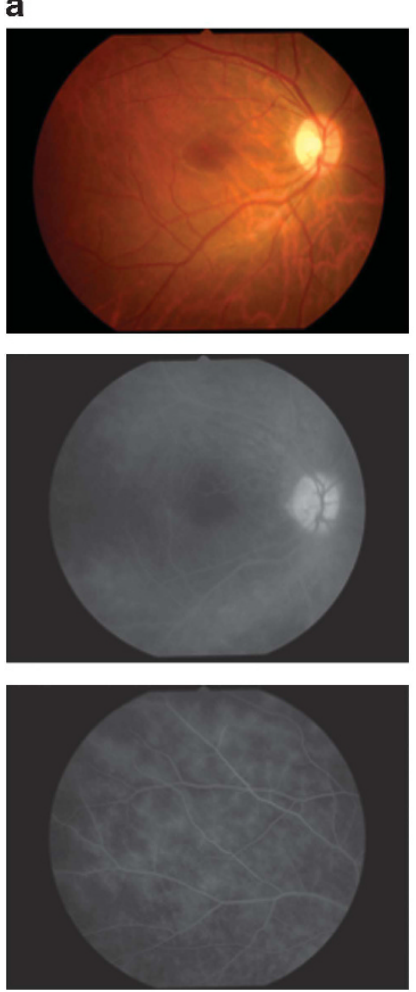

b
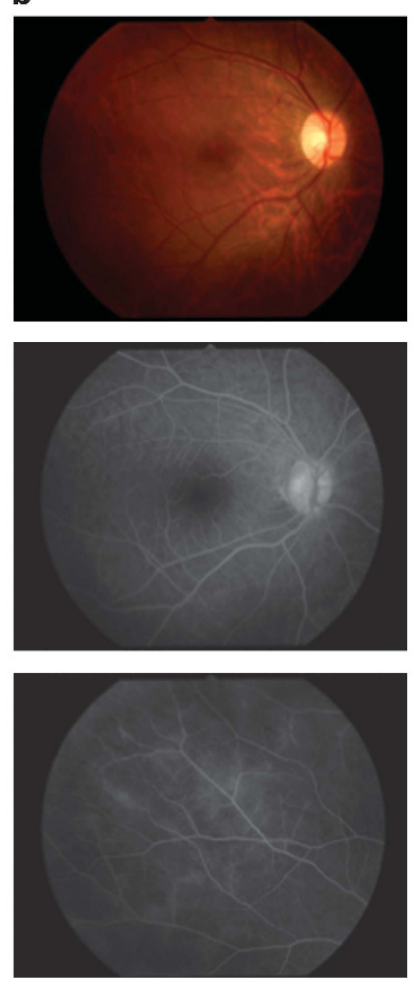

c
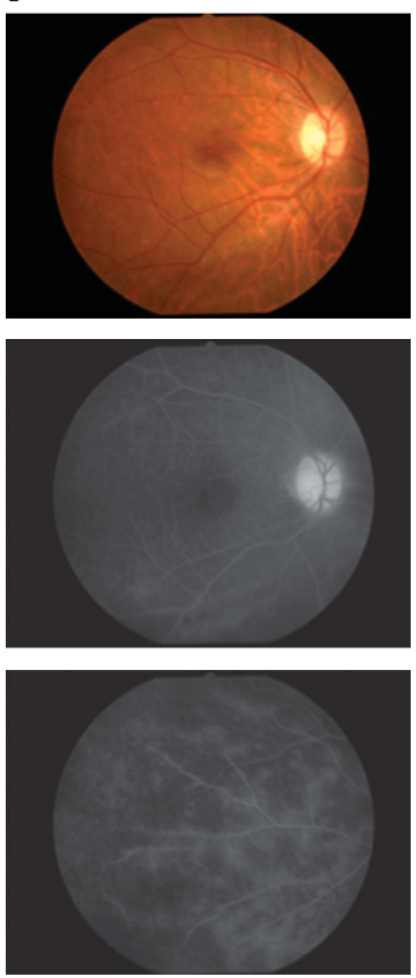

Figure 3 Color fundus photographs and FA images of patient 5 at baseline and on infliximab therapy. Color fundus photographs and FA images are shown for the right eye of patient 5. All FA examinations were performed during times of clinical quiescence. (a) Fluorescein leakage from vessels at the optic disc, macula, and peripheral retina was observed before infliximab treatment (left upper and lower images). The vascular leakage score was 4.5 at this time (baseline). (b) Fluorescein leakage improved on infliximab therapy at the 6-month time point (middle upper and lower images). The vascular leakage score was 2.0. (c) However, FA leakage appeared to have increased at the end of 4 years (right upper and lower images) compared with at 6 months. The vascular leakage score was 4.0 . 
from 150 to $125 \mathrm{mg}$ /day without recurrence of ocular attacks. As shown in Figure $3 a$ and $b$ (baseline and 6 months, respectively), improvement in vascular leakage from the disc, macula, and peripheral retina was initially observed. However, the patient began to experience mild attacks in both eyes after 6 months. Thus, the interval between infliximab infusions was shortened from 8 to 6 weeks; however, the patient continued to have mild ocular attacks. As shown in Figure 3c, FA vascular leakage from the disc, macula, and peripheral retina was worse at the end of 4 years compared with at 6 months.

\section{Patient 7}

This patient was a 35-year-old Japanese man who was diagnosed in June 2006 with recurrent uveoretinitis due to Behçet's disease. Despite treatment with cyclosporine, the patient had posterior ocular attacks in both eyes, and infliximab therapy was started in February 2008.

Although the patient had two mild ocular attacks over the first year after initiation of infliximab treatment, no recurrences were observed subsequently through the end of 4 years. Color fundus photographs and FA images are shown in Figure 4a (baseline) and Figure $4 \mathrm{~b}$ (end of year 3), there was decreased vascular leakage from the disc, macula, and peripheral retina.

\section{Discussion}

In the current study, infliximab therapy significantly reduced the mean frequency of ocular inflammatory attacks in Behçet's disease patients over a long-term

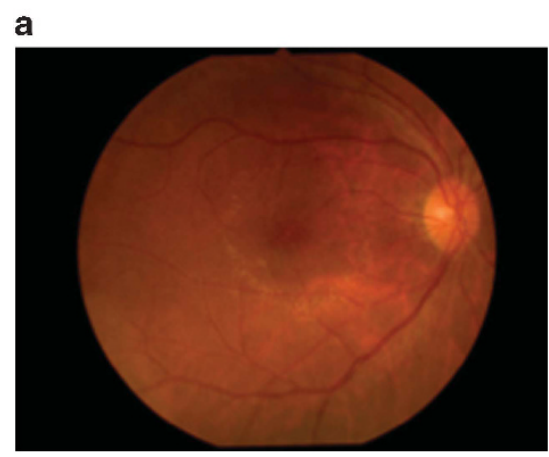

b
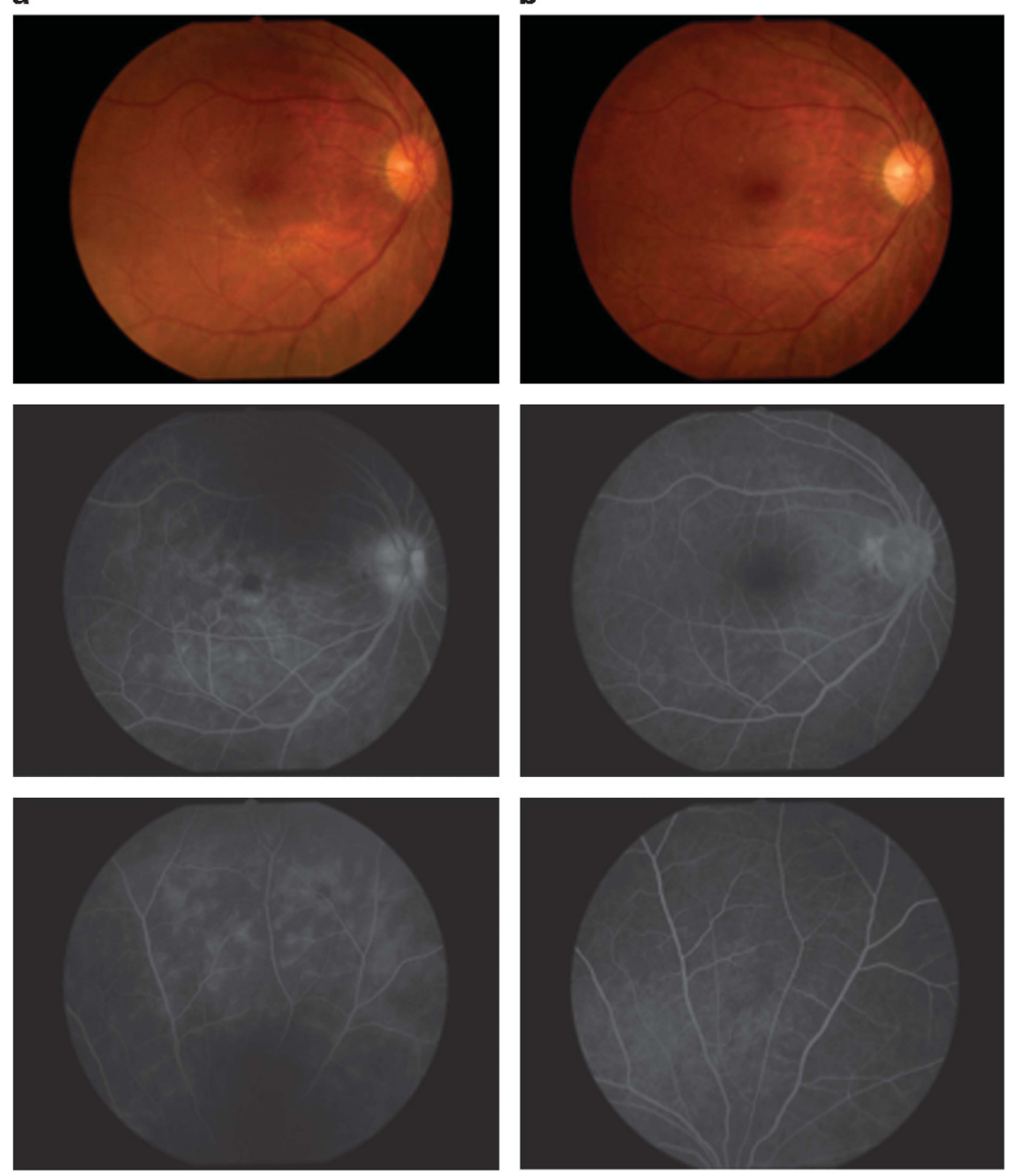

Figure 4 Color fundus photographs and FA images of patient 7 at baseline and on infliximab therapy. Color fundus photographs and FA images are shown for the right eye of patient 7. All FA examinations were performed during times of clinical quiescence. (a) Fluorescein leakage from vessels at the optic disc, macula, and peripheral retina was observed before initiating infliximab therapy (left upper and lower images). The vascular leakage score was 5 at this time (baseline). (b) This fluorescein leakage improved after 3 years on infliximab therapy (right upper and lower images). The vascular leakage score was 0.5. 
follow-up of 4 years. In addition, the mean total vascular leakage score also significantly decreased at the end of years 1,2,3, and 4 of infliximab therapy when compared with baseline, and the BCVAs were maintained or improved in 17 of 18 eyes at the end of 4 years. No serious adverse effects were observed during the followup period in any patients. These results suggest that infliximab is effective in a fairly sustained manner, and has a relatively good safety profile, over the long period of 4 years in Behçet's disease patients with refractory uveoretinitis. Furthermore, this study confirms what has already been reported for Behçet's disease patients with shorter term follow-up on infliximab. ${ }^{19-21}$

Recently, we have shown that infliximab therapy significantly reduced retinal vascular leakage scores at 12 months compared with the baseline. ${ }^{14}$ In the present study, we confirmed the sustained effect of infliximab on suppressing background vascular leakage over 4 years, although it took $\sim 2$ years for vascular leakage scores to reach their lowest level. Although a recent study has shown the rapid effect of infliximab in suppressing acute ocular inflammation associated with Behçet's disease, ${ }^{22}$ improvement in the barrier function of retinal and disc vessels may require longer periods of infliximab treatment.

Reduction in frequency of ocular attacks and FA vascular leakage score was maintained over the 4-year period in the majority of patients. However, patient 5 experienced frequent mild attacks after 12 months and the total vascular leakage score showed mild trend toward rising after 24 months despite shortening of infusion intervals from 8 to 6 weeks. This suggests a degree of acquired resistance to infliximab in this patient. Similar acquired resistance after 12 months of infliximab treatment in Behçet's disease patients has been reported. ${ }^{23}$ In an attempt to overcome such resistance, infusions may be administered more frequently as we have done, or the dose of infliximab infused at each time may be increased. The latter is not approved by the MHLW and thus, our only option was to increase the frequency of infusions. It is well known that some patients treated with infliximab develop antibodies against the drug, often referred to as HACA. ${ }^{16}$ Development of HACA has been reported to be associated with low circulating drug levels. ${ }^{24}$ Although we have not measured the serum levels of infliximab in our patients, it is possible that the levels may be lower at the end of 4 years compared with earlier in the course of infliximab treatment. More frequent infusions and/or greater doses of infliximab may be required to elevate infliximab serum levels in some Behçet's disease patients suspected of having acquired resistance.

In the majority of our patients, low-dose cyclosporine was continued as concomitant immunosuppressive therapy with the hopes of reducing the immunogenicity of infliximab. In patients with rheumatoid arthritis, the use of infliximab with concomitant methotrexate has been shown to reduce the incidence of HACA and also reduce the frequency of infusion reactions, ${ }^{16}$ although it is unclear whether reduction in infusion reactions correlates with better efficacy. At this time, it is also unclear whether concomitant immunosuppressive therapy is capable of suppressing the production of HACA in Behçet's disease patients, nor which immunosuppressive agent is most effective for this purpose. A recent multicenter study demonstrated the efficacy of infliximab for refractory uveoretinitis in Behçet's disease during the first year of treatment in Japan, ${ }^{13}$ and a follow-up study examining the influence of concomitant immunosuppressive agents on infliximab efficacy is being conducted in this cohort.

Limitations of our study include that the data was examined retrospectively, the number of patients was small, and all patients were from a single institution. However, our study suggests that infliximab is safe and effective in a sustained manner over a long period of time. Infliximab suppressed background retinal and optic disc vascular leakage, decreased ocular attacks, and maintained or improved BCVA in Behçet's disease patients with refractory uveoretinitis treated for at least 4 years with infliximab.

\section{What was known before \\ - Infliximab is an effective therapy for uveoretinitis in Behcet's disease patients. Only short-term efficacy and safety have been reported with the use of infliximab.}

\section{What this study adds}

- This study reports long-term results and safety of infliximab treatment. Long-term data validate infliximab as an effective and safe treatment for reducing ocular inflammatory attacks, as well as background retinal and disc vascular leakage in Behcet's disease patients.

\section{Conflict of interest}

This study was supported by restricted research funds from Mitsubishi Tanabe Pharma for the Department of Ophthalmology, Kyorin University School of Medicine.

\section{References}

1 Mochizuki M, Akduman L, Nussenblatt RB. Behcet's disease. In: Pepose JS, Holland GN, Wilhelmus KR (eds). Ocular Infection and Immunity. Mosby: St Louis, MO, USA, 1996, pp 663-675.

2 Nussenblatt RB. Uveitis in Behcet's disease. Int Rev Immunol 1997; 14: 67-79.

3 Yates PA, Michelson JB. Behcet's disease. Int Ophthalmol Clin 2006; 46: 209-233. 
4 Evereklioglu C. Current concepts in the etiology and treatment of Behcet's disease. Surv Ophthalmol 2005; 50: 297-350.

5 Kaburaki T, Araki F, Takamoto M, Okinaga K, Yoshida A, Numaga J et al. Best-corrected visual acuity and frequency of ocular attacks during the initial 10 years in patients with Behçet's disease. Graefes Arch Clin Exp Ophthalmol 2010; 248: 709-714.

6 George RK, Chan CC, Whitcup SM, Nussenblatt RB. Ocular immunopathology of Behçet's disease. Surv Ophthalmol 1997; 42: 157-162.

7 Atmaca LS. Fundus changes associated with Behcet's disease. Graefes Arch Clin Exp Ophthalmol 1989; 227: 340-344.

8 Okada AA. Behcet's disease: general concepts and recent advances. Curr Opin Ophthalmol 2006; 17: 551-556.

9 Ohno S, Nakamura S, Hori S, Shimakawa M, Kawashima H, Mochizuki $\mathrm{M}$ et al. Efficacy, safety, and pharmacokinetics of multiple administration of infliximab in Behcet's disease with refractory uveoretinitis. J Rheumatol 2004; 31: 1362-1368.

10 Tugal-Tutkun I, Mudun A, Urgancioglu M, Kamali S, Kasapoglu E, Inanc M et al. Efficacy of infliximab in the treatment of uveitis that is resistant to treatment with the combination of azathioprine, cyclosporine, and corticosteroids in Behcet's disease. Arthritis Rheum 2005; 52: 2478-2484.

11 Yamada Y, Sugita S, Tanaka H, Kamoi K, Kawaguchi T, Mochizuki M.. Comparison of infliximab versus ciclosporine during the initial 6-month treatment period in Behcet's disease. Br J Ophthalmol 2010; 94: 284-288.

12 Yamada Y, Sugita S, Tanaka H, kamoi K, Takase H, Mochizuki M. Timing of recurrent uveitis in patients with Behçet's disease receiving infliximab treatment. $\mathrm{Br} \mathrm{J}$ Ophthalmol 2011; 95: 205-208.

13 Okada AA, Goto H, Ohno S, Mochizuki M. Multicenter study of infliximab for refractory uveoretinitis in Behcet's disease. Arch Ophthalmol 2012; 130: 592-598.

14 Keino H, Okada AA, Watanabe T, Taki W. Decreased ocular inflammatory attacks and background retinal and disc vascular leakage in patients with Behçet's disease on infliximab therapy. Br J Ophthalmol 2011; 95: 1245-1250.

15 Mizushima Y. The Revised Criteria and Manual of Treatments of Behcet's disease. In: Report of Behcet's Disease
Research Committee. Ministry of Health and Welfare of Japan: Tokyo, Japan, 1987, pp 16-17.

16 Maini RN, Breedveld FC, Kalden JR, Smolen JS, Davis D, Macfarlane JD et al. Therapeutic efficacy of multiple intravenous infusions of anti-tumor necrosis factor alpha monoclonal antibody combined with low-dose weekly methotrexate in rheumatoid arthritis. Arthritis Rheum 1998; 41: 1552-1563.

17 Baert F, Noman M, Vermeire S, Van Assche G, D Haens G, Carbonez A et al. Influence of immunogenicity on the long-term efficacy of infliximab in Crohn's disease. $\mathrm{N} \mathrm{Engl} \mathrm{J}$ Med 2003; 348: 601-608.

18 Jabs DA, Nussenblatt RB, Rosenbaum JT. The Standardization of Uveitis Nomenclature (SUN) Working Group. Standardization of uveitis nomenclature for reporting clinical data. Results of the First International Workshop. Am J Ophthalmol 2005; 140: 509-516.

19 Abu El-Asrar AM, Abboud EB, Aldibhi H, Al-Arfaj A. Long-term safety and efficacy of infliximab therapy in refractory uveitis due to Behçet's disease. Int Ophthalmol 2005; 26: 83-92.

20 Niccoli L, Nannini C, Benucci M, Chindamo D, Cassarà E, Salvarani $\mathrm{C}$ et al. Long-term efficacy of infliximab in refractory posterior uveitis of Behçet's disease: a 24-month follow-up study. Rheumatology 2007; 46: 1161-1164.

21 Handa T, Tsunekawa H, Yoneda M, Watanabe D, Mukai T, Yamamura $\mathrm{M}$ et al. Long-term remission of ocular and extraocular manifestations in Behçet's disease using infliximab. Clin Exp Rheumatol 2011; 29: S58-S63.

22 Markomichelakis N, Delicha E, Masselos S, Fragiadaki J, Kaklamanis P, Sfikakis PP. A single infliximab infusion vs corticosteroids for acute panuveitis attacks in Behçet's disease: a comparative 4-week study. Rheumatology 2011; 50: 593-597.

23 Ito T, Sonoda KH, Hijioka K, Fujimoto T, Ishibashi T. Acquired resistance to infliximab against uveitis due to Behçet's disease after one year of administration. Jpn J Ophthalmol 2010; 54: 502-504.

24 Svenson M, Geborek P, Saxne T, Bendtzen K. Monitoring patients treated with anti-TNF- $\alpha$ biopharmaceuticals: assessing serum infliximab and anti-infliximab antibodies. Rheumatology 2007; 46: 1828-1834. 